\title{
SOCIAL ADAPTATION OF PATIENTS WITH CHRONIC SKIN DISEASES
}

\author{
Drumeva P. ${ }^{1}$, S. Bachvarova ${ }^{2}$, R. Bachvarova ${ }^{3}$ \\ ${ }^{1}$ Clinic of Dermatovenereology, ${ }^{2}$ Clinic of Neurology and Psychiatry, Department of Clinical Medical \\ Sciences, and ${ }^{3}$ Department of Psychiatry and Medical Psychology, Medical University of Varna
}

Reviewed by: Assoc. Prof. R. Shiskov, MD, PhD

\begin{abstract}
A Social Adaptation Self-Evaluation Scale (SAAS) was applied to follow-up the social adaptation of 61 patients with skin diseases, 32 males and 29 females aged between 21 and 68 years occasionally examined in the outpatient consulting room of the Clinic of Dermatovenereology at the Medical University of Varna. The results vary within the limits of slight to moderate aberrations mainly when assessing the outer appearance, interrelations, contacts and expressions in the society as well as concerning the satisfaction with the work process. The familial relationships which compensate to a certain extent the other disturbances prove to be most harmonious.
\end{abstract}

Keywords: chronic skin disease, social adaptation, Social Adaptation Self-Evaluation Scale, quality of life, family relationships

\section{INTRODUCTION}

Usually, the patients with skin diseases are the object of outpatient observation. Thus they are seldom admitted to hospital and most exacerbations are treated under outpatient conditions as well. That is why their milieu does not change. Some investigations prove however, that in a considerable number of these patients there exist anxiety, depression and social dysadaptation. These disturbances are directly related to the localization and the extent of dissemination of skin lesions (3-5). Shah and Coates (2006) assessed dermatological patients' quality of life by the Dermatology Life Quality Index, the Hospital Anxiety and Depression Scale and the Illness Perception Questionnaire. The severity of the psoriasis of the patients was measured using the Psoriasis Area and Severity Index, Dermatology Life Quality Index and Psoriasis Disability Index (2). The quality of life (QoL) of the relatives and partners was more closely related to the patients' QoL than to the objective disease severity scores (2). Visible cosmetic defects lead more easily to the loss of numerous social interests and reduction of patient's quality of life.

The purpose of the present study is to follow-up the degree of social dysadaptation of the patients with chronic skin diseases located on the visible part of the body who are within their normal society and follow their usual life rhythm.

\section{MATERIAL AND METHODS}

We used a cross-sectional study design. It covered a total of 61 patients, 32 males and 29 females aged between 21 and 68 years. They presented with chronic skin diseases affecting the open parts of the body. These patients had occasionally undergone medical examinations in the outpatient consulting room of the Clinic of Dermatovenereology at the Medical University of Varna. The patients with psychotic diseases, organic disorders of the central nervous system, psychoactive-drug abuse (i. e., of narcotics, alcohol, and medicines) as well as with severe somatic diseases were excluded from the study.

A Social Adaptation Self-Evaluation Scale (SAAS) (1) was applied. It consists of 21 questions dealing with the leisure, social contacts, work, enlarged family, and public life. Every question is scored by $0-3$ scores corresponding to the minimal up to the maximal adaptation. According to the responses, the total sum of the scores remains within the interval from 0 to 60 scores.

The results obtained allowed to differentiate five groups of patients ranging from perfectly adapted subjects (score one) to socially affected ones (score five). The span between 35 and 52 scores was considered a standard one. The questionnaire was filled-in under anonymous conditions in order to avoid additional psychotraumatic harmful agents and thus to obtain more objective results.

Thirty healthy individuals matched according to age and social status who had visited the outpatient examination room on the occasion of issuing their medical certificates concerning jobs, arms and ammunition, and marriage in St. Marina Diagnostic and Consulting Centre of Varna served as controls.

\section{RESULTS AND DISCUSSION}

The mean parameters of SAAS demonstrate a value of $31,97 \%$ while in the control group, these amount to $47,8 \%$ $(\mathrm{p}<0,05)$. This argues to the presence of a slight 
dysadaptation. Usually, the parameters vary within slight to moderate aberrations from the normal values. This fact can be explained as these patients are not disabled yet and their life-style is comparatively adequate to the contemporary standards. On the other hand, however, the evaluation of the structure of the results demonstrates that the patients experience definite troubles in certain fields of the social life while some compensatory mechanisms are developed in the other areas to neutralize some components of the anomalies.

The worries about the outer appearance are most outlined what is psychologically determined by the characteristics of the diseases. It is noteworthy, however, that significant gender differences are established only in this respect, indeed. The males present with a higher percentage (of $81 \%$ ) than the females (of 60\%) although during the clinical examination nobody of the male patients has complained of this worry to the treating physician.

The attempts to create and maintain the interrelations with people outside the family are particularly hampered (in $78 \%$ of the cases) along with the issues related to the quality of such communication (in 59\%). Because of these reasons, probably, some patients ( $49 \%$ of the cases) do not consider the informal contacts as a priority and respond that the interrelations with other people have been either of "little importance", or of "certain importance only" for them. It is likely that the aforementioned disturbances and the nature of the pathological manifestations reflect upon the activity and participation of the patients under examination in the social life (in 59\% of the cases). Besides they feel troubles to express their own opinion at places that are socially significant for them (in 30\% of the cases). Parallel with that, possibly as compensation, their social curiosity is at an extraordinarily high level (89\%). Nevertheless, some patients (69\% of the cases) share that the quality of their leisure time remains at a low level and thus it is not interesting at all ( $80 \%$ of the cases). These results indicate that skin affections lead to intervertedness, they restrict or even hinder the new relations and interrelationships, minimize the old ones and thus crate a peculiar isolation and capsulation of the patient into a narrower circle of friends. In this way, they considerably reduce the level and quality of the informal social contacts and the expression of the people of this category in public life. In a more distant respect, these results are suspicious of the presence of anxiety or depression disturbances. The analysis of the data about the experiences within the working environment demonstrates interesting tendencies, too. On the one hand, the attitude to work of most respondents $(68,85 \%$ of the cases) is positive and they consider their job very and moderately interesting. There does not exist, however, any correlation between these emotions and the experienced satisfaction with the labour process itself, as $40,98 \%$ of the respondents do their job with certain or little pleasure. In this respect, it can be supposed that skin problems affect, if even only indirectly, the quality of work and work capacity of the individual with skin disease.

The interrelations within the enlarged family seem to be most harmonious, indeed. Almost all the persons $(76,6 \%$ of the cases) render an account that they often look for contacts with their husbands/wives, children, parents and relatives. Besides they score the quality of these relationships very high. When analyzing these results we should obligatorily take into consideration the peculiar Bulgarian's ethnopsychology as alienation typical of Western civilization is much more seldom here. In our opinion, however, certain defence mechanisms are involved, too, that could not be excluded and, probably, compensate the adaptation disturbances caused by the skin diseases.

A link between the mind and the skin has long been hypothesized. Conditions such as demoralization, health anxiety, irritable mood, type A behaviour, and alexithymia were also found to be frequent in dermatological patients, and to be independently associated with greater psychological distress, lower quality of life, and poorer psychosocial functioning (7). The diagnostic criteria for psychosomatic research have been found to yield valuable integrative information, in addition to DSM-IV nosology, in a variety of medical dermatological diseases (6).

\section{CONCLUSION}

We could draw the conclusion that although we have performed only a cross-sectional observation of the influence of the chronic skin diseases located on the visible part of the body on social life we establish the fact that these diseases are capable of disturbing the freedom of the relationships and contacts, expressions at public places, the qualitative utilization of leisure time as well as, to a certain extent, the pleasure of the work done. Independently of the harmony of the familial relationships which partially minimize these unpleasant manifestations, a dysadaptation definitively exists with these patients.

The results from our pilot investigation allow us to plan a future research aiming at continuing the more detailed study of this issue by a dynamical following-up the severity of the social dysadaptation according to the diagnosis of the skin disease, patient's gender and degree of dissemination of the skin affections as well as by looking for concrete mechanisms for its improvement.

\section{REFERENCES}

1. Bosc, M., A. Dubini, V. Polin. Development and validation of a social functioning scale, the Social Adaptation Self-Evaluation Scale.- Eur.

Neuropsyhopharmacol., 7, 1997, 57-70.

2. Eghlileb, A.M., E. E. Davies, A. Y. Finlay. Psoriasis has a major secondary impact on the lives of family members and partners.- Br. J. Dermatol., 156, 2007, 1245-1250.

3. Gupta, M., A. Gupta. Depression and suicidal ideation in dermatology patients with acne alopecia areata, atopic dermatitis and psoriasis.- $B r . J$. Dermatol., 139, 1998, 846-850.

4. Milard, L. Dermatological practice and psychiatriy.Br. J. Dermatol., 143, 2000, 920-921. 
5. Picardi, A., D. Abeni, C. Melichi, et al. Psychiatric morbidity in dermatological outpatients: an issue to be recognized.- Br. J. Dermatol., 143, 2000, 983-991.

6. Picardi, A., P. Porcelli, P. Pasquini, et al. Integration of multiple criteria for psychosomatic assessment of dermatological patients.- Psychosomatics, 47, 2006, 122-128.
7. Picardi, A., P. Pasquini. Toward a biopsychosocial approach to skin diseases.- Adv. Psychosom. Med., 28, 2007, 109-126.

8. Shah, M., M. Coates. An assessment of the quality of life in older patients with skin disease.- $B r . J$. Dermatol., 154, 2006, 150-153. 